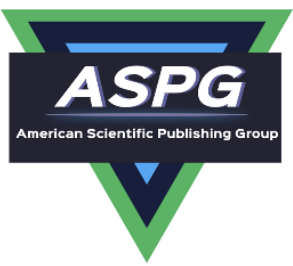

\title{
There is No Constant in Physics: a Neutrosophic Explanation
}

\author{
Victor Christianto ${ }^{1}$, Robert N. Boyd ${ }^{2}$ and Florentin Smarandache ${ }^{3, *}$ \\ ${ }^{1}$ Satyabhakti Advanced School of Theology, Jakarta-Chapter, INDONESIA; victorchristianto@gmail.com \\ 2 Consulting Physicist at Princeton Biotechnology Corporation, Princeton, USA; rnboydphd@comcast.net \\ 3 Dept. Math and Sciences, University of New Mexico, Gallup, NM, USA; smarand@unm.edu \\ * Correspondence: victorchristianto@gmail.com
}

\begin{abstract}
In Neutrosophic Logic, a basic assertion is that there are variations of about everything that we can measure; the variations surround three parameters called T,I,F (truth, indeterminacy, falsehood) which can take a range of values. Similarly, in this paper we consider NL applications in physics constants. Those constants actually all have a window of plus and minus values, relative to the average value of the constant. For example, the window for the speed of light is approximately: $+3000 \mathrm{~m} / \mathrm{s}>\mathrm{c}<$ minus $3000 \mathrm{~m} / \mathrm{s}$. We also discuss some implications of this new perspective of physics constants, including in gravitation physics etc.
\end{abstract}

Keywords: Neutrosophic Logic, Physical Neutrosophy, gravitation, physics constants

\section{Introduction}

For majority of physicists, constants play a fundamental role. Like an anchor for a ship, they allow physicists build theories on the ground of those constants as basic "known" quantities. However, in real experiments, there are always variation of those constants. Moreover, from Neutrosophic Logic perspective, those constants always fluctuate depending on various circumstances.

In Neutrosophic Logic, a basic assertion is that there are variations of about everything that we can measure, the variations surround three parameters called T,I,F (truth, indeterminacy, falsehood) which can take a range of values. Similarly, in this paper we consider NL applications in physics constants. Those constants actually all have a window of plus and minus values, relative to the average value of the constant. For example, the window for the speed of light is approximately: $+3000 \mathrm{~m} / \mathrm{s}>\mathrm{c}<$ minus $3000 \mathrm{~m} / \mathrm{s}$. We also discuss some implications of this new perspective of physics constants, including in gravitation physics etc.

It is our hope that this new perspective on physics constants will point to a more substantial and evidence-based approach to physics sciences.

\section{Definition}

Neutrosophic Logic, as developed by one of us (FS), is generalization of fuzzy logic based on Neutrosophy. A proposition is $\mathrm{t}$ true, $\mathrm{i}$ indeterminate, and $\mathrm{f}$ false, where $\mathrm{t}, \mathrm{i}$, and $\mathrm{f}$ are real values from the ranges $\mathrm{T}$, I, F, with no restriction on $\mathrm{T}, \mathrm{I}, \mathrm{F}$, or the sum $\mathrm{n}=\mathrm{t}+\mathrm{i}+\mathrm{f}$. Neutrosophic logic thus generalises: 
- intuitionistic logic, which supports incomplete theories (for $0<\mathrm{n}<100$ and $\mathrm{i}=0,0<=\mathrm{t}, \mathrm{i}, \mathrm{f}<=100$ );

- fuzzy logic (for $\mathrm{n}=100$ and $\mathrm{i}=0$, and $0<=\mathrm{t}, \mathrm{i}, \mathrm{f}<=100$ );

- Boolean logic (for $\mathrm{n}=100$ and $\mathrm{i}=0$, with $\mathrm{t}, \mathrm{f}$ either 0 or 100 );

- multi-valued logic (for $0<=\mathrm{t}, \mathrm{i}, \mathrm{f}<=100$ );

- paraconsistent logic (for $\mathrm{n}>100$ and $\mathrm{i}=0$, with both $\mathrm{t}, \mathrm{f}<100$ );

- dialetheism, which says that some contradictions are true ( $\mathrm{for} \mathrm{t}=\mathrm{f}=100$ and $\mathrm{i}=0$; some paradoxes can be denoted this way).

Compared with all other logics, neutrosophic logic introduces a percentage of "indeterminacy" - due to unexpected parameters hidden in some propositions. It also allows each component t,i,f to "boil over" 100 or "freeze" under 0. For example, in some tautologies $t>100$, called "overtrue".[1]

Neutrosophic Logic allows one to develop new approaches in many fields of science, including a redefinition of physics constants, as will be discussed in the next section.

\section{Neutrosophic reasoning: There is no Physics Constant}

In accordance with Neutrosophic Logic, actually all physics constants have a window of plus and minus values, relative to the average value of the constant. For example, the window for the speed of light is approximately: $+3000 \mathrm{~m} / \mathrm{s}>\mathrm{c}<$ minus $3000 \mathrm{~m} / \mathrm{s}$.

There may be larger excursions, but we would not expect larger excursions to happen very often. Probability considerations are thus also involved in determining the average value and the statistical extremes for the given constant.

There are also curves which vary according to the materials involved, and the environment. For example, most recently and most importantly, it has been realized that $\mathrm{h}$ and $\mathrm{h}$ _bar cannot be used for any material other than carbon black (soot).

All other materials must have their thermal emissions curve instrumented. Then the $\mathrm{h}$ and $\mathrm{h} \_$bar for that material can be calculated. But the values calculated are subject to modifications by the local environment. Unless the aether environment can be considered and measured, the calculated values of $h$ and $h$ bbar for the given material will not be as reliable as we might prefer. (It depends on the specific application which requires instrumented measurements of the thermal emissions curve of the given material.)

So there should be a way to produce an accurate thermal emissions curve using a neutrosophic approach. Because all thermal emissions curves have extremes from absolute zero to very high heat values. Neutrosophic modifications of Kirchoff's law of "blackbody radiation", and Planck's "constant" would be very useful. (See for instance, a report by Robitaille and Crothers on the flaws of Kirchoff law, [2-4]). It is worth noting here, that from dynamical perspective, Shpenkov argues for a redefinition of Planck constant: "The Planck constant $h$ is the quantity the value of which is equal to the orbital action of the electron on the Bohr first orbit in the hydrogen atom, namely to its orbital moment of momentum $\mathrm{P}_{\text {orb }}$ multiplied by $2 \pi$, or it can be rewritten as: $h=2 \pi$. $P_{\text {orb }}$." According to him, Planck constant also has acoustic origin. [5]

There are also physical situations where the variations of the value of one constant, directly alters the values of physically-related constants. The fine structure "constant" is an example of this kind of mutual influence. If the fine structure value changes, it changes the value of e, the charge of the electron. (Which informs us that the charge on 
the electron is an environmentally influenced Neutrosophic window.) Going the other way, if the value of e changes, it changes the value of the fine structure constant.

Another aspect of this to consider is that some constants-windows may not be perfectly symmetrical, but large on one side of the center value, and small on the other side, and exhibit dependence on the environment, such that under most conditions the value of the given "constant" would live inside the window, while there could be large asymmetrical extremes at other times, depending on the local and non-local environmental parameters of the aether, at the location where we are examining the measured value of the constant.

\section{A few applications}

At this point, some readers may ask: Can we get an example when a so-called constant has a value, while in another example the same so-called constant gets another value?

Answer: Gravitation is a good example. g changes depending on where and when it is measured. This is used in gravitational prospecting and by the GRACE experiment (NASA) which maps the gravitation variations of the Earth, over time. [6] In ref [6], they show many more data sets and graphical images showing gravitational variations on the Earth.

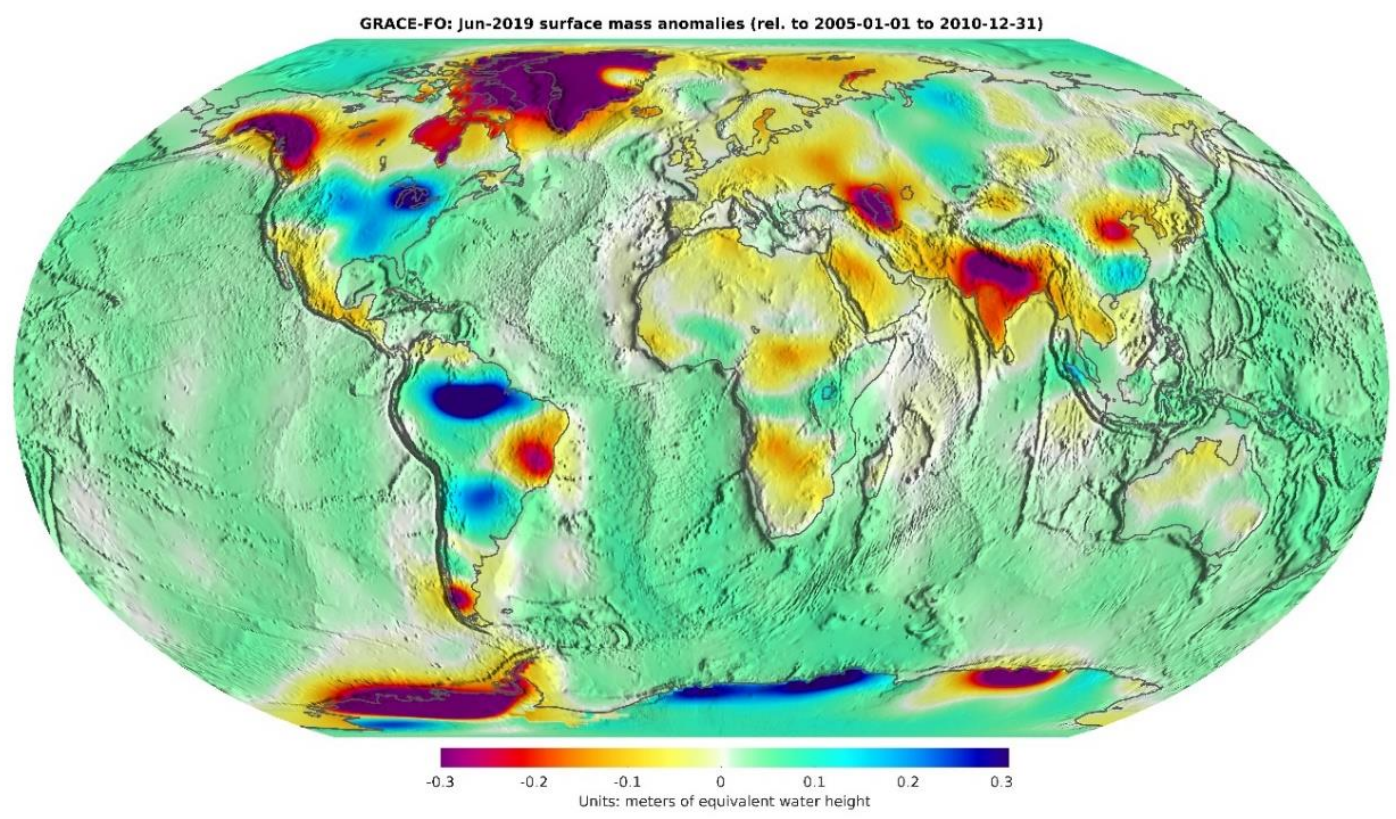

Figure 1. gravitational variations on the Earth. Source: [6]

Another article contains a good table of measurements of gravitation from 1798, until 2004 [7].

There is also a discussion of the increase in the force due to gravitation of the Earth, showing the dinosaurs would be crushed by their own weight if they were subjected to the gravitational force of the Earth today.

The gravitational "constant" is a good one to start with, since the variations can't be denied.

The next best one would be speed of light variations, although these days they refuse to allow one-way measurements of light velocity, because vast numbers of variations show up, depending on the time and place of the measurement. The mainstream insists that the speed of light can only be measured by round-trip measurements. This 
is because the light going back and forth along the same line results in many of the measured one-way variations in the velocity, being averaged out.

Typically, speed of light experiments cook their books and throw out any large deviations in measured light velocity. This tactic is similar to even more egregious cheating methods which are used by "global warming" and "climate change" advocates, paid for by the oil companies.

The next best one would be variations in Planck's "constant". $\mathrm{h}$ and $\mathrm{h} \_$bar are only valid for carbon black (soot). Every different material has a different thermal radiation when plotted on a thermal radiation curve. Some examples are displayed by Robitaille and Crothers in some of their presentations on the original "black body" thermal radiation constant known as Kirchoff's law, which was never measured by instrumented experiments, and was accepted as universally valid by Planck, who never did experiments to measure the thermal radiation curves of anything.[2-4]

\section{Conclusions}

In this article, we discussed how physics constants can vary in a wide range of values, in particular from Neutrosophic Logic perspective. We also discussed some examples, including variation in Earth gravitation measurements, speed of light measurement, and also Planck constant. It is our hope that this short discussion will be found as good impetus for a new direction in physics, more corresponding to experimental data, toward: "evidencebased physics." This new direction is in direct contrast to the unfortunate development of theoretical physics in the last 30-40 years with their overreliance on too much abstraction, oversophisticated mathematics, and other fantasies, which often have less and less to do with the actual physics as an empirical science. Two books can be mentioned here in relation to the present situation of physics science, see [8][9].

Funding: "This research received no external funding"

Conflicts of Interest: "The authors declare no conflict of interest."

\section{References}

1. F. Smarandache. Neutrosophy / Neutrosophic probability, set, and logic, American Research Press, 1998. See also: http://gallup.unm.edu/ smarandache/NeutLog.txt.

2. P-M. Robitaille; S. Crothers. "The Theory of Heat Radiation" Revisited: A Commentary on the Validity of Kirchhoff's Law of Thermal Emission and Max Planck's Claim of Universality, Prog. Phys. Vol. 11 issue 2 (2015). url: www.ptep-online.com. See also: http://vixra.org/pdf/1502.0007v1.pdf.

3. P-M. Robitaille. A Re-examination of Kirchhoff's Law of Thermal Radiation in Relation to Recent Criticisms: Reply. Prog. Phys., 2016, v. 12, no. 3, 184-203. url: www.ptep-online.com. See also: http://www.vixra.org/abs/1602.0004

4. R.J. Johnson, A Re-examination of Kirchhoff's Law of Thermal Radiation in Relation to Recent Criticisms. Prog. Phys., 2016, v.12, no.3, 175-18.

5. G. P. Shpenkov, On the Fine-Structure Constant : Physical Meaning, Hadronic Journal Vol. 28, No. 3, 337-372, (2005). url: https://shpenkov.com/pdf/Fine-Structure.pdf

6. https://grace.jpl.nasa.gov/

7. http://blazelabs.com/f-u-massvariation.asp

8. R. Penrose. Fashion, Faith and Fantasy in the new Physics of the Universe. Princeton: Princeton University Press, 2017. Url: https://press.princeton.edu/books/hardcover/9780691119793/fashion-faith-and-fantasy-in-thenew-physics-of-the-universe

9. S. Hossenfelder. Lost in Math: How beauty leads physics astray. Basic Books, 2018. url: https://www.amazon.com/Lost-Math-Beauty-Physics-Astray/dp/0465094252 